\title{
HARMONIC TORI IN QUATERNIONIC PROJECTIVE 3-SPACES
}

\author{
SEIICHI UDAGAWA
}

(Communicated by Peter Li)

\begin{abstract}
Burstall classified conformal non-superminimal harmonic two-tori in spheres and complex projective spaces. In this paper, we shall classify conformal non-superminimal harmonic two-tori in a 2- or 3-dimensional quaternionic projective space, which are not always covered by primitive harmonic two-tori of finite type.
\end{abstract}

\section{INTRODUCTION}

The harmonic two-spheres in $n$-dimensional quaternionic projective space $\mathbf{H} P^{n}$ are studied and classified in [Ba-W]. In contrast with the case of harmonic twospheres, there is a class of non-conformal harmonic maps for two-torus. For nonconformal harmonic two-tori in compact symmetric space of rank one, a beautiful theory is established by [B-F-P-P], which says that they are obtained by integrating certain commuting Hamiltonian flows. They called the map of this kind a map of finite type. However, the geometrically interesting class of harmonic maps is that of conformal ones. The (weakly) conformal harmonic maps are, again, divided into two subclasses, the class of superminimal ones and the class of non-superminimal ones. The former class is well understood and it is obtained by projecting a horizontal holomorphic map into a certain generalized flag manifold.

In [B], Burstall proved that any non-superminimal harmonic torus in a sphere or a complex projective space is covered by a primitive harmonic map of finite type into a certain generalized flag manifold. In the previous paper [U], the present author generalized Burstall's result to $G_{2}\left(\mathbf{C}^{4}\right)$ as target. Moreover, for the general complex Grassmann manifold as target, some sufficient conditions for a given harmonic map be of finite type are given (see [U]).

In the present paper, we treat the harmonic two-tori in quaternionic projective space. We prove the classification theorem for non-superminimal harmonic two-tori in $\mathbf{H} P^{2}$ and $\mathbf{H} P^{3}$ (Theorems 5.1 and 5.2).

\section{Quaternionic PRojeCtive SPaCe and harmonic SeQuence}

Let $\mathbf{C}^{2 n}$ be a $2 n$-dimensional complex number space with the standard Hermitian inner product $\langle$,$\rangle defined by \langle v, w\rangle=\sum_{i=1}^{2 n} v_{i} \overline{w_{i}}$, where $v=\left(v_{1}, v_{2}, \cdots, v_{2 n}\right), w=$ $\left(w_{1}, w_{2}, \cdots, w_{2 n}\right)$. Let $\mathbf{H}$ be the division ring of quaternions and $j$ a unit quaternion with $j^{2}=-1$. The correspondence $\mathbf{C}^{2} \ni\left(v_{1}, w_{1}\right) \longrightarrow v_{1}+w_{1} j \in \mathbf{H}$ yields an identification $\mathbf{C}^{2} \cong \mathbf{H}$. In general, we have an identification $\mathbf{C}^{2 n} \cong \mathbf{H}^{n}$. Let

Received by the editors June 26, 1995.

1991 Mathematics Subject Classification. Primary 58E20, 53C42. 
$J: \mathbf{C}^{2 n} \longrightarrow \mathbf{C}^{2 n}$ be the conjugate linear map given by left multiplication by $j$, that is,

$$
J\left(v_{1}, v_{2}, \cdots, v_{2 n}\right)=\left(-\overline{v_{2}}, \overline{v_{1}}, \cdots,-\overline{v_{2 n}}, \overline{v_{2 n-1}}\right) .
$$

Then, $J^{2}=-I$, where $I$ is the identity map on $\mathbf{C}^{2 n}$. Let $G_{2}\left(\mathbf{C}^{2 n}\right)$ be the Grassmann manifold of all complex 2-dimensinal subspaces of $\mathbf{C}^{2 n}$ with its standard Kähler structure. The quaternionic projective space $\mathbf{H} P^{n-1}$ may be regarded as the totally geodesic submanifold of $G_{2}\left(\mathbf{C}^{2 n}\right)$ as follows :

$$
\mathbf{H} P^{n-1}=\left\{V \in G_{2}\left(\mathbf{C}^{2 n}\right) \mid V=J V\right\},
$$

that is, the set of all complex 2-dimensional subspaces of $\mathbf{C}^{2 n}$ which are closed under the action of $J$. The inner product $\langle$,$\rangle has the following property :$

$$
\langle J v, J w\rangle=\overline{\langle v, w\rangle}=\langle w, v\rangle \quad \text { for all } v, w \in \mathbf{C}^{2 n} .
$$

Let $\varphi: M \longrightarrow G_{2}\left(\mathbf{C}^{2 n}\right)$ be a smooth map of a Riemann surface. Let $V(\varphi)$ be the pull-back of universal bundle over $G_{2}\left(\mathbf{C}^{2 n}\right)$ by $\varphi$. Then, $V(\varphi)$ is a subbundle of the trivial bundle $V\left(\mathbf{C}^{2 n}\right)=M \times \mathbf{C}^{2 n}$. We equip $V\left(\mathbf{C}^{2 n}\right)$ with the standard Hermitian connected structure compatible with the Hermitian metric $\langle$,$\rangle . For any$ subbundle $F$ of $V\left(\mathbf{C}^{2 n}\right)$, we denote by $F^{\perp}$ the Hermitian orthogonal complement of $F$ in $V\left(\mathbf{C}^{2 n}\right)$ with respect to $\langle$,$\rangle . Then, F$ and $F^{\perp}$ are both equipped with the Hermitian connected structures induced from that of $V\left(\mathbf{C}^{2 n}\right)$. Moreover, $F$ and $F^{\perp}$ both have the Koszul-Malgrange holomorphic structures. Let $A_{1}^{F, F^{\perp}}$ be the $(1,0)$-part of the second fundamental form of $F$ in $V\left(\mathbf{C}^{2 n}\right)$. By taking the image of the second fundamental form, we may define the new subbundle $F_{1}$ of $V\left(\mathbf{C}^{2 n}\right)$, which is defined on $M$ except the singularity subset $S$. If $A_{1}^{F, F^{\perp}}$ is a holomorphic section, $S$ is a discrete set. In this case, the line bundle $[S]$ defined by the divisor $S$ enables us to extend $F_{1}$ smoothly over $M$, which is also denoted by $F_{1}$. Set $V_{0}=$ $V(\varphi)$. It is known that $A_{1}^{V_{0}, V_{0}^{\perp}}$ is a $\operatorname{Hom}\left(V_{0}, V_{0}^{\perp}\right)$-valued holomorphic differential if and only if $\varphi$ is a harmonic map. It is also known that $V_{1}$ defines a harmonic map $\varphi_{1}: M \longrightarrow G_{k}\left(\mathbf{C}^{2 n}\right)$ with $k \leq 2$, where $V_{1}$ is isomorphic to the pull-back of the universal bundle over $G_{k}\left(\mathbf{C}^{2 n}\right)$ by $\varphi_{1}$. Repeating this procedure, we obtain the harmonic sequence $V_{0} \rightarrow V_{1} \rightarrow V_{2} \rightarrow \cdots$ of the bundles or the harmonic map sequence $\left\{\varphi_{i}\right\}$. In the case where we use $(0,1)$-part of the second fundamental form $A_{\prime \prime}^{F, F^{\perp}}$, we denote the corresponding harmonic sequence by $V_{0} \leftarrow V_{-1} \leftarrow V_{-2} \leftarrow \cdots$ (see $[\mathrm{Ba}-\mathrm{W}, \mathrm{B}-\mathrm{W}, \mathrm{W}])$.

Definition. A harmonic map $\varphi$ is said to have strong isotropy order $r$ if $V_{0} \perp V_{i}$ for $i=1, \cdots, r$ and $V_{r+1}$ is not perpendicular to $V_{0}$ with respect to $\langle$,$\rangle . In the$ case of $r=\infty, \varphi$ is said to be strongly isotropic or superminimal.

Finally, we prepare the following lemma.

Lemma 1.1. For any subbundle $F$ of $V\left(\mathbf{C}^{2 n}\right)$, we have

$$
\left(A_{,}^{J F,(J F)^{\perp}} \circ J\right)(v)=\left(J \circ A_{\prime \prime}^{F, F^{\perp}}\right)(v), \quad\left(A_{\prime \prime}^{J F,(J F)^{\perp}} \circ J\right)(v)=\left(J \circ A_{,}^{F, F^{\perp}}\right)(v),
$$

where $v \in C^{\infty}(F)$.

Proof. Let $\partial^{\prime}$ and $\partial^{\prime \prime}$ be the $(1,0)$ - and $(0,1)$-differentiations, respectively, of the flat connection on $V\left(\mathbf{C}^{2 n}\right)$. Then, since $J$ is a conjugate linear map, we have 
$\partial^{\prime} \circ J=J \circ \partial^{\prime \prime}$ and $\partial^{\prime \prime} \circ J=J \circ \partial^{\prime}$. If we denote by $\nabla^{E}$ the connection of the bundle $E$, for $v \in C^{\infty}(F)$ we have

$$
\begin{aligned}
& \partial^{\prime}(J v)=\nabla_{\prime}^{J F} J v+A_{\prime}^{J F,(J F)^{\perp}}(J v), \\
& J\left(\partial^{\prime \prime} v\right)=J \nabla_{\prime \prime}^{F} v+J A_{\prime \prime}^{F, F^{\perp}}(v),
\end{aligned}
$$

where $\nabla_{\prime}^{E}$ and $\nabla_{\prime \prime}^{E}$ are the $(1,0)$ - and $(0,1)$-part of $\nabla^{E}$, respectively. Since we see that $(J F)^{\perp}=J\left(F^{\perp}\right)$ by using (1.1), comparing the components of the two equations, we obtain the first desired equation. Similarly, using the equation $\partial^{\prime \prime} \circ J=J \circ \partial^{\prime \prime}$ we obtain the second desired equation.

\section{HARMONIC MAPS OF FINITE TYPE}

Let $G$ be a compact semisimple Lie group. Let $N=G / K$ be a reductive homogeneous space. We have the reductive decomposition of the Lie algebra $\mathcal{G}$ of $G$ :

$$
\mathcal{G}=\mathcal{K}+\mathcal{M}, \quad[\mathcal{K}, \mathcal{M}] \subset \mathcal{M}
$$

where $\mathcal{K}$ is the Lie algebra of $K$ and $\mathcal{M}$ is identified with the tangent space $T_{o} N$ of $N$ at the base point $o$. Suppose that there is an (inner) automorphism $\tau: \mathcal{G} \rightarrow \mathcal{G}$ of order $k$ with fixed set $\mathcal{K}$. Set $\zeta=\exp (2 \pi \sqrt{-1} / k)$. Then, the complexification $\mathcal{G}^{\mathbf{C}}$ of $\mathcal{G}$ is decomposed as

$$
\mathcal{G}^{\mathbf{C}}=\sum_{i \in \mathbf{Z}_{k}} \mathcal{G}_{i}
$$

where $\mathcal{G}_{i}$ is the $\zeta^{i}$-eigenspace of $\tau$. We have

$$
\begin{aligned}
\mathcal{M}^{\mathbf{C}} & =\sum_{i=1}^{k-1} \mathcal{G}_{i}, & \mathcal{K}^{\mathbf{C}}=\mathcal{G}_{0}, \\
\overline{\mathcal{G}}_{i} & =\mathcal{G}_{-i} & {\left[\mathcal{G}_{i}, \mathcal{G}_{j}\right] \subset \mathcal{G}_{i+j} }
\end{aligned}
$$

The map $\mathcal{G} \rightarrow T_{x} N$ given by $\left.\xi \rightarrow \frac{d}{d t}\right|_{t=0} \exp t \xi \cdot x$ restricts to an isomorphism $\operatorname{Ad} g \mathcal{M} \rightarrow T_{x} N$. We denote the inverse map by $\beta: T_{x} N \rightarrow \operatorname{Ad} g \mathcal{M} \subset \mathcal{G}$ and we may regard $\beta$ as a $\mathcal{G}$-valued 1-form on $N$, which is called Maurer-Cartan form of $N$ (see [B-R]). In this situation, $N$ becomes a $k$-symmetric space in the sense of Kowalski $([\mathrm{K}])$. Define the bundle $\left[\mathcal{G}_{i}\right]$ by $\left[\mathcal{G}_{i}\right]_{x}=\operatorname{Ad} g \cdot \mathcal{G}_{i}$ for $x=g \cdot o$.

Let $\psi: M \longrightarrow N$ be a smooth map of a Riemann surface.

Definition. $\psi$ is said to be a primitive map if $\psi^{*} \beta(\partial / \partial z)$ is $\left[\mathcal{G}_{1}\right]$-valued.

In the case of $k=2$, the primitive map condition is meaningless because $\mathcal{M}^{\mathrm{C}}=$ $\mathcal{G}_{1}$. For $k>2$, any primitive map $\psi$ is a harmonic $\operatorname{map}([\mathrm{B}, \mathrm{B}-\mathrm{P}])$. Let $F: M \longrightarrow G$ be a (local) lift of $\psi: M \longrightarrow N$ with projection given by $F \rightarrow F \cdot o$. Such $F$ always exists locally and is called a framing of $\psi$.

Now, suppose that $G$ is a matrix group. Set $\alpha=F^{-1} d F$. Write $\alpha=\alpha_{\mathcal{K}}+\alpha_{\mathcal{M}}$ according as the decomposition $\mathcal{G}=\mathcal{K}+\mathcal{M}$. Write also $\alpha_{\mathcal{M}}=\alpha_{\mathcal{M}}^{\prime}+\alpha_{\mathcal{M}}^{\prime \prime}$, where $\alpha_{\mathcal{M}}^{\prime}$ and $\alpha_{\mathcal{M}}^{\prime \prime}$ are the $(1,0)$ - and $(0,1)$-forms with values in $\mathcal{M}$, respectively. We have

$$
\psi^{*} \beta=\operatorname{Ad} F \alpha_{\mathcal{M}}
$$


Now, $\psi$ is a primitive map if and only if $\alpha_{\mathcal{M}}^{\prime}(\partial / \partial z)$ is $\mathcal{G}_{1}$-valued. We give examples of certain primitive maps, the construction of which is due to Burstall ([B]). We fix an Iwasawa decomposition :

$$
\mathcal{K}^{\mathbf{C}}=\mathcal{K} \oplus \mathcal{B}
$$

where $\mathcal{B}$ is a solvable subalgebra of $\mathcal{K}^{\mathbf{C}}$. Such a decomposition exists since $\mathcal{K}$ is compact so that $\mathcal{K}^{\mathbf{C}}$ is reductive.

Set

$$
\wedge \mathcal{G}_{\tau}^{\mathbf{C}}=\left\{\xi: S^{1} \longrightarrow \mathcal{G}^{\mathbf{C}} \mid \xi(\zeta \lambda)=\tau \xi(\lambda) \text { for } \lambda \in S^{1}\right\},
$$

which is an infinite dimensional Lie algebra. We equip it with the Sobolev $H^{r}$ topology for some $r>1 / 2$. Let $\wedge \mathcal{G}_{\tau}$ be the real form

$$
\wedge \mathcal{G}_{\tau}=\left\{\xi \in \wedge \mathcal{G}_{\tau}^{\mathbf{C}} \mid \xi: S^{1} \longrightarrow \mathcal{G}\right\} .
$$

Any $\xi \in \wedge \mathcal{G}_{\tau}$ has a Fourier expansion $\xi=\sum \xi_{n} \lambda^{n}$. Define a finite dimensional subspace $\wedge_{d}$ as follows :

$$
\wedge_{d}=\left\{\xi \in \wedge \mathcal{G}_{\tau} \mid \xi_{n}=0 \text { for all }|n|>d\right\} .
$$

Let $d \equiv 1 \bmod k$. Then, $\xi_{d} \in \mathcal{G}_{1}$ and $\xi_{d-1} \in \mathcal{K}^{\mathbf{C}}$. Let $\mathcal{T}$ be the given maximal torus in $\mathcal{K}$ and $\mathcal{N}$ the nilpotent subalgebra given by the positive root spaces and set $\mathcal{H}=\mathcal{T}^{\mathbf{C}}$. Then, we have

$$
\mathcal{K}^{\mathbf{C}}=\mathcal{N} \oplus \mathcal{H} \oplus \overline{\mathcal{N}} \quad, \quad \mathcal{B}=(\sqrt{-1} \mathcal{T}) \oplus \mathcal{N} .
$$

Any element $\eta \in \mathcal{K}^{\mathbf{C}}$ may be written as $\eta=\eta_{\mathcal{N}}+\eta_{\mathcal{H}}+\eta_{\mathcal{N}}$. Define a map $r: \mathcal{K}^{\mathbf{C}} \rightarrow$ $\mathcal{K}^{\mathbf{C}}$ by

$$
r(\eta)=\eta_{\overline{\mathcal{N}}}+\frac{1}{2} \eta_{\mathcal{H}}
$$

(see $[\mathrm{B}-\mathrm{P}]$ ). Now, take a $\xi_{0} \in \wedge_{d}$ as an initial condition and solve the differential equation

$$
\frac{\partial \xi}{\partial z}=\left[\xi, \lambda \xi_{d}+r\left(\xi_{d-1}\right)\right] ; \quad \xi(0)=\xi_{0} .
$$

Then, there is a primitive harmonic map $\psi: \mathbf{R}^{2} \longrightarrow N$ with framing $F: \mathbf{R}^{2} \longrightarrow G$ satisfying $F^{-1} \partial F / \partial z=\xi_{d}+r\left(\xi_{d-1}\right)$.

Definition. A primitive harmonic map $\psi$ obtained by solving the eqaution (2.4) is said to be of finite type.

We have a question : "What kind of primitive harmonic maps are of finite type?" There is the following answer:

Theorem 2.1 ([B-F-P-P, B-P, B]). Let $\psi: T^{2} \longrightarrow N$ be a primitive harmonic map of a two-torus into a $k$-symmetric space $(k \geq 2)$. Suppose that $\psi^{*} \beta(\partial / \partial z)$ is semisimple on a dense subset of $T^{2}$. Then, $\psi$ is of finite type.

Now, let $p: G / K \longrightarrow G / H$ be the homogeneous projection with $K \subset H$ and $\psi: M \longrightarrow G / K$ be a primitive harmonic map of a Riemann surface. Then, it is known that $p \circ \psi: M \longrightarrow G / H$ is also a harmonic map ([B-P, B]). Next, we consider the question: "What kind of harmonic map into $G_{k}\left(\mathbf{C}^{n}\right)$ is covered by a primitive harmonic map into $N$ ?" Let $\varphi: M \longrightarrow G_{k}\left(\mathbf{C}^{n}\right)$ be a non-superminimal harmonic map of a Riemann surface with strong isotropy order $r$. Set $V_{0}=V(\varphi)$ and let $V_{0} \rightarrow V_{1} \rightarrow \cdots \rightarrow V_{r-1} \rightarrow V_{r}$ be the harmonic sequence of the bundles 
for $\varphi$, where each $V_{i}$ is of rank $k$ for $i=0,1, \cdots, r-1$ and $\operatorname{rank} V_{r}=n-r k$. Set $G=S U(n)$. Let $N=S U(n) / S(U(k) \times \cdots \times U(k) \times U(n-r k))$ be the flag manifold. Any point $x$ of $N$ may be expressed as $x=\left(w_{0}, w_{1}, \cdots, w_{r}\right)$, where $w_{i}$ is a $k$-plane for $i=0,1, \cdots, r-1$ and $w_{r}$ is an $(n-r k)$-plane. Let $p: N \longrightarrow G_{k}\left(\mathbf{C}^{n}\right)$ be the projection map which assigns to the flag its first element ; $p\left(w_{0}, w_{1}, \cdots, w_{r}\right)=w_{0}$. Fix any point $x=\left(w_{0}, w_{1}, \cdots, w_{r}\right) \in N$ and define $Q \in G$ by

$$
Q=\zeta^{i} \text { on } w_{i} \text { for } i=0,1, \cdots, r,
$$

where $\zeta=\exp (2 \pi \sqrt{-1} / r+1)$. Then, $\tau=\operatorname{Ad} Q$ is an order $(r+1)$ automorphism of $G$, and the identity component of its fixed set is conjugate to $S(U(k) \times \cdots \times U(k) \times$ $U(n-r k))$, which we denote by $K$. Thus, $N=G / K$ becomes an $(r+1)$-symmetric space (see $[\mathrm{K}]$ ). We define a map $\psi: M \longrightarrow N=G / K$ by

$$
\psi(p)=\left(\left(V_{0}\right)_{p},\left(V_{1}\right)_{p}, \cdots,\left(V_{r}\right)_{p}\right) \text { for } p \in M .
$$

Then, $\varphi=p \circ \psi$. It turns out that such $\psi$ is necessarily a primitive map (Lemma $3.1 \mathrm{in}[\mathrm{U}])$.

Finally, we consider the question: "What kind of harmonic map into $G_{k}\left(\mathbf{C}^{n}\right)$ is covered by a primitive harmonic map of finite type?" Let $A_{\gamma_{\varphi}}^{F R}$ be the $\partial^{\prime}$-first return map for $\varphi$, that is,

$$
A_{l \varphi}^{F R}=A_{1}^{V_{r}, V_{0}} \circ A_{,}^{V_{r-1}, V_{r}} \circ \cdots \circ A_{1}^{V_{0}, V_{1}} .
$$

We have the following answer:

Theorem $2.2([\mathrm{U}])$. Let $\varphi: T^{2} \longrightarrow G_{k}\left(\mathbf{C}^{n}\right)$ be a harmonic map. If the $\partial^{\prime}$-first return map $A_{1 \varphi}^{F R}$ for $\varphi$ is semisimple and invertible on a dense subset of $T^{2}$, then $\varphi$ is covered by a primitive harmonic map of finite type into $S U(n) / S(U(k) \times \cdots \times$ $U(k) \times U(n-r k))$, where $r$ is the strong isotropy order of $\varphi$.

For example, any non-superminimal harmonic tori in $\mathbf{C} P^{n-1}$ and any weakly conformal non-superminimal harmonic tori in $G_{2}\left(\mathbf{C}^{4}\right)$ may be classified using Theorem 2.2 (see $[\mathrm{U}])$.

\section{HARMONIC MAPS OF ODD STRONG ISOTROPY ORDER}

In this section, we investigate the $\partial^{\prime}$-first return map for harmonic maps of odd strong isotropy order.

Let $\varphi: M \longrightarrow \mathbf{H} P^{n-1} \subset G_{2}\left(\mathbf{C}^{2 n}\right)$ be a non-superminimal harmonic map with strong isotropy order $r$. Suppose that $r$ is odd. Let $A_{1 \varphi}^{F R}$ and $A_{/ / \varphi}^{F R}$ be the $\partial^{\prime}$ - and $\partial^{\prime \prime}$-first return map for $\varphi$, respectively. As in (2.5), the $\partial^{\prime \prime}$ - first return map is defined by

$$
A_{1 / \varphi}^{F R}=A_{\| \prime}^{V_{-r}, V_{0}} \circ A_{\| \prime}^{V_{-r+1}, V_{-r}} \circ \cdots \circ A_{\| \prime}^{V_{0}, V_{-1}} .
$$

Let $\left\{e_{1}, J e_{1}\right\}$ be a local unitary basis for $V_{0}$. Then, using (1.1) and Lemma 1.1 we have

$$
\begin{aligned}
\left\langle A_{l \varphi}^{F R}\left(e_{1}\right), J e_{1}\right\rangle & =-\left\langle e_{1}, J A_{\prime \varphi}^{F R}\left(e_{1}\right)\right\rangle \\
& =-\left\langle e_{1}, A_{{ }^{\prime \prime \varphi}}^{F R}\left(J e_{1}\right)\right\rangle \\
& =-(-1)^{r+1}\left\langle A_{l \varphi}^{F R}\left(e_{1}\right), J e_{1}\right\rangle .
\end{aligned}
$$


Since $r$ is odd, we obtain $\left\langle A_{\iota_{\varphi}}^{F R}\left(e_{1}\right), J e_{1}\right\rangle=0$. Similarly, we have $\left\langle A_{l_{\varphi}}^{F R}\left(J e_{1}\right), e_{1}\right\rangle=$ 0 . Again, using (1.1) and Lemma 1.1 we obtain

$$
\begin{aligned}
\left\langle A_{/ \varphi}^{F R}\left(J e_{1}\right), J e_{1}\right\rangle & =\left\langle J A_{/ / \varphi}^{F R}\left(e_{1}\right), J e_{1}\right\rangle \\
& =\left\langle e_{1}, A_{/ / \varphi}^{F R}\left(e_{1}\right)\right\rangle \\
& =(-1)^{r+1}\left\langle A_{l_{\varphi}}^{F R}\left(e_{1}\right), e_{1}\right\rangle .
\end{aligned}
$$

Since $r$ is odd, we obtain $\left\langle A_{/ \varphi}^{F R}\left(J e_{1}\right), J e_{1}\right\rangle=\left\langle A_{/ \varphi}^{F R}\left(e_{1}\right), e_{1}\right\rangle$. Therefore, the $\partial^{\prime}$ - first return map for $\varphi$ is of the form

$$
A_{\iota \varphi}^{F R}=\left(\begin{array}{cc}
a & 0 \\
0 & a
\end{array}\right)
$$

where $a$ is non-zero on a dense subset of $M$ because $\varphi$ is non-superminimal. This, together with Theorem 2.2, yields :

Proposition 3.1. Let $\varphi: T^{2} \longrightarrow \mathbf{H} P^{n-1}$ be a non-superminimal harmonic map of strong isotropy order $r$. Suppose that $r$ is odd. Then, $\varphi$ is covered by a primitive harmonic map of finite type into $S U(2 n) / S(U(2) \times \cdots \times U(2) \times U(2 n-2 r))$.

\section{HARMONIC MAPS OF EVEN STRONG ISOTROPY ORDER}

In this section, we investigate the $\partial^{\prime}$ - first return map for harmonic maps of even strong isotropy order.

Let $\varphi: M \longrightarrow \mathbf{H} P^{n-1} \subset G_{2}\left(\mathbf{C}^{2 n}\right)$ be a non-superminimal harmonic map with strong isotropy order $r$. Suppose that $r$ is even.

Let $\left\{e_{1}, J e_{1}\right\}$ be a local unitary basis for $V_{0}$. Then, by (3.2) we obtain

$$
\operatorname{trace} A_{l \varphi}^{F R}=0 .
$$

Therefore, by (4.1) and Theorem 2.2 we have :

Proposition 4.1. If $\operatorname{det} A_{1 \varphi}^{F R} \neq 0$ on a dense subset of $T^{2}$, then $\varphi$ is covered by a primitive harmonic map of finite type into $S U(2 n) / S(U(2) \times \cdots \times U(2) \times U(2 n-2 r))$.

Thus, in the following, we consider the case where $A_{1 \varphi}^{F R}$ is nilpotent. Set $B=$ $\operatorname{Im} A_{1 \varphi}^{F R}$, which is extended over $M$ and a holomorphic line subbundle of $V_{0}$. Since $A_{1 \varphi}^{F R}$ is nilpotent, $B \subset \operatorname{Ker} A_{1 \varphi}^{F R}$. Then, we obtain

Lemma 4.1. Define $\varphi_{1}$ by the forward replacement of $B$ from $\varphi$, that is,

$$
V\left(\varphi_{1}\right)=V(\varphi) \ominus B \oplus \operatorname{Im}\left(\left.A_{1}^{V(\varphi), V(\varphi)^{\perp}}\right|_{B}\right) .
$$

Then, $\varphi_{1}$ has strong isotropy order $r+1$.

Proof. Set $B_{0}=B$ and $C_{0}=V(\varphi) \ominus B$. Define $B_{i}$ for $i=1, \cdots, r$ by $B_{i}=$ $\operatorname{Im}\left(\left.A_{,}^{V_{i-1}, V_{i}}\right|_{B_{i-1}}\right)$. Set $C_{i}=V_{i} \ominus B_{i}$ for $i=1, \cdots, r-1$. Set $V_{r}^{\prime}=\operatorname{Im} A_{\prime}^{V_{r-1}, V_{r}}, C_{r}=$ $V_{r}^{\prime} \ominus B_{r}$ and $R=V_{r} \ominus V_{r}^{\prime}$. By the nilpotency of $A_{\ell_{\varphi}}^{F R}$, we see that $\left.A_{r}^{V_{r}^{\prime}, V_{r}^{\prime \perp}}\right|_{B_{r}}$ has images in $R$ and is a holomorphic bundle map. Hence, we set $B_{r+1}=\operatorname{Im}\left(\left.A_{1}^{V_{r}^{\prime}, V_{r}^{\prime \perp}}\right|_{B_{r}}\right)$ and $R^{\prime}=R \ominus B_{r+1}$. We want to show that $A_{1}^{B_{r+1}, C_{0}} \equiv 0$. Then, $\varphi_{1}$ has strong isotropy order $r+1$ (see [B-W]). Suppose the contrary. We denote by $\left\{V_{n}\left(\varphi_{1}\right)\right\}(n \in \mathbf{Z})$ the harmonic sequence of the bundles for $\varphi_{1}$. We have $V_{-1}\left(\varphi_{1}\right) \subset$ $\left(B_{0} \oplus B_{r+1} \oplus R^{\prime}\right)$. Then, we see that

$$
V_{-\frac{r}{2}-1}\left(\varphi_{1}\right) \cap V_{\frac{r}{2}}\left(\varphi_{1}\right)=B_{\frac{r}{2}+1} .
$$


On the other hand, since $V_{-1}\left(\varphi_{1}\right)=J V\left(\varphi_{1}\right)$, using Lemma 1.1 we obtain

$$
J V_{\frac{r}{2}}\left(\varphi_{1}\right)=V_{-\frac{r}{2}-1}\left(\varphi_{1}\right),
$$

which, together with (4.2), implies that $J V_{\frac{r}{2}}\left(\varphi_{1}\right) \cap V_{\frac{r}{2}}\left(\varphi_{1}\right)=B_{\frac{r}{2}+1}$, which is impossible because the $J$-invariant subspace must be even-dimensional. Therefore, we may conclude that $A_{1}^{B_{r+1}, C_{0}} \equiv 0$ and thus $\varphi_{1}$ has strong isotropy order $r+1$.

A harmonic map $\varphi_{1}: M \longrightarrow G_{2}\left(\mathbf{C}^{2 n}\right)$ is no longer quaternionic. We want to search for an anti-holomorphic line subbundle $\delta$ of $V\left(\varphi_{1}\right)$ so that the backward replacement of $\varphi_{1}$ by $\delta$ may give a harmonic map of strong isotropy order at least $r+1$ which has values in quaternionic projective space $\mathbf{H} P^{n-1} \subset G_{2}\left(\mathbf{C}^{2 n}\right)$. The existence of such a line subbundle is ensured for the harmonic two-spheres in $\mathbf{H} P^{n-1}$ (see $[\mathrm{Ba}-\mathrm{W}]$ ). For the harmonic two-tori in $\mathbf{H} P^{n-1}$, it is not necessarily ensured. However, we have the following

Proposition 4.2. Let $A_{1 \varphi_{1}}^{F R}$ be the $\partial^{\prime}$-first return map for $\varphi_{1}$ and $\left\{J e_{1}, e_{1}^{1}\right\}$ a local unitary basis for $V\left(\varphi_{1}\right)$. Define a line subbundle $\delta$ of $V\left(\varphi_{1}\right)$ locally by

$$
\delta=\operatorname{Span}_{\mathbf{C}}\left\{-\left\langle A_{\prime \prime}^{\varphi_{1}}\left(J e_{1}\right), e_{1}\right\rangle e_{1}^{1}+2\left\langle A_{\prime \prime}^{\varphi_{1}}\left(e_{1}^{1}\right), e_{1}\right\rangle J e_{1}\right\} .
$$

Then, $\delta$ is an anti-holomorphic line subbundle of $V\left(\varphi_{1}\right)$, which may be extended over $M$. Define a harmonic map $\varphi_{2}$ by the backward replacement of $\delta$ from $\varphi_{1}$. If $\left(\operatorname{trace} A_{l_{1}}^{F R}\right)^{2}-4 \operatorname{det}\left(A_{l_{1}}^{F R}\right)=0$, then $\varphi_{2}$ has strong isotropy order greater than or equal to $r+1$ and is quaternionic.

Proof. First of all, we investigate the form of the $\partial^{\prime}$ - first return map for $\varphi_{1}$. Since $\varphi_{1}$ has strong isotropy order at least $r+1$ by Lemma 4.1 , we denote by $\left\{V_{i}\right\}_{i=0}^{r+1}$ its harmonic sequence of the bundles. Set

$$
A_{\prime \prime}^{V_{0}, V_{r+1}}\left(e_{1}^{1}\right)=\beta e_{1} .
$$

Then, we may set

$$
A_{1}^{V_{r+1}, V_{0}}\left(e_{1}\right)=-\bar{\beta} e_{1}^{1}+s J e_{1} .
$$

Note that $\beta$ is non-zero on a dense subset of $M$. From (4.5) we have

$$
A_{r}^{V_{r+1}, V_{0}} \circ A_{1}^{V_{r}, V_{r+1}} \circ \cdots \circ A_{1}^{V_{0}, V_{1}} \circ A_{,}^{V_{r+1}, V_{0}}\left(e_{1}\right)=-\bar{\beta} A_{\varphi_{1}}^{F R}\left(e_{1}^{1}\right)+s A_{\varphi_{1}}^{F R}\left(J e_{1}\right) .
$$

Taking the inner product of this equation with $e_{1}^{1}$ and using (4.4), we obtain

$$
\left\langle A_{/ V_{r+1}}^{F R}\left(e_{1}\right), e_{1}\right\rangle=\left\langle A_{\varphi_{1}}^{F R}\left(e_{1}^{1}\right), e_{1}^{1}\right\rangle-\frac{s}{\bar{\beta}}\left\langle A_{l_{1}}^{F R}\left(J e_{1}\right), e_{1}^{1}\right\rangle
$$

where $A_{/ V_{r+1}}^{F R}$ is the $\partial^{\prime}$-first return map for $V_{r+1}$. On the other hand, using (1.1) and Lemma 1.1 we have

$$
\begin{aligned}
\left\langle A_{\iota_{1}}^{F R}\left(J e_{1}\right), J e_{1}\right\rangle & =\left\langle J A_{1 / V_{r+1}}^{F R}\left(e_{1}\right), J e_{1}\right\rangle \\
& =\left\langle A_{V_{r+1}}^{F R}\left(e_{1}\right), e_{1}\right\rangle,
\end{aligned}
$$

which, together with (4.6), implies that the $\partial^{\prime}$-first return map for $\varphi_{1}$ is of the form

$$
A_{1 \varphi_{1}}^{F R}=\left(\begin{array}{cc}
a & b \\
c & a-\frac{s}{\beta} c
\end{array}\right)
$$

where $a=\left\langle A_{\varphi_{1}}^{F R}\left(e_{1}^{1}\right), e_{1}^{1}\right\rangle, b=\left\langle A_{\varphi_{1}}^{F R}\left(e_{1}^{1}\right), J e_{1}\right\rangle$ and $c=\left\langle A_{\varphi_{1}}^{F R}\left(J e_{1}\right), e_{1}^{1}\right\rangle$. Note that $c$ is non-zero on a dense subset of $M$ by the definition of $V\left(\varphi_{1}\right)$. That $\delta$ defined in (4.3) is an anti-holomorphic subbundle of $V\left(\varphi_{1}\right)$ follows from the fact that $A_{\prime \prime}^{\varphi_{1}}$ is 
an anti-holomorphic section. Now, $\delta=\operatorname{Span}_{\mathbf{C}}\left\{\bar{s} e_{1}^{1}+2 \beta J e_{1}\right\}$ and $\operatorname{Im}\left(\left.A_{\| \prime}^{V_{0}, V_{r+1}}\right|_{\delta}\right)$ $=\operatorname{Span}_{\mathbf{C}}\left\{\bar{s} e_{1}+2 \beta J e_{1}^{1}\right\}$. Then, $J \operatorname{Im}\left(\left.A_{\prime \prime}^{V_{0}, V_{r+1}}\right|_{\delta}\right)=\operatorname{Span}_{\mathbf{C}}\left\{s J e_{1}-2 \bar{\beta} e_{1}^{1}\right\}$, which is orthogonal to $\delta$ with respect to $\langle$,$\rangle and is contained in V\left(\varphi_{1}\right)$. Therefore, we see that $V\left(\varphi_{2}\right)$ is quaternionic. To investigate the strong isotropy order of $\varphi_{2}$, we want to know whether

$$
\left\langle A_{1}^{V_{r}, V_{r+1}} \circ \cdots \circ A_{\prime}^{V_{0}, V_{1}}\left(J \operatorname{Im}\left(A_{\prime \prime}^{V_{0}, V_{r+1}} \mid \delta\right)\right), \operatorname{Im}\left(A_{\prime \prime}^{V_{0}, V_{r+1}} \mid \delta\right)\right\rangle
$$

is zero or not. Using (4.4), (4.5) and Lemma 1.1, we calculate

$$
\begin{aligned}
& \left\langle A_{1}^{V_{r}, V_{r+1}} \circ \cdots \circ A_{1}^{V_{0}, V_{1}}\left(s J e_{1}-2 \bar{\beta} e_{1}^{1}\right), \bar{s} e_{1}+2 \beta J e_{1}^{1}\right\rangle \\
& =s^{2}\left\langle A_{1}^{V_{r}, V_{r+1}} \circ \cdots \circ A_{1}^{V_{0}, V_{1}}\left(J e_{1}\right), e_{1}\right\rangle+2 \bar{\beta} s\left\langle A_{1}^{V_{r}, V_{r+1}} \circ \cdots \circ A_{1}^{V_{0}, V_{1}}\left(J e_{1}\right), J e_{1}^{1}\right\rangle \\
& -2 \bar{\beta} s\left\langle A_{,}^{V_{r}, V_{r+1}} \circ \cdots \circ A_{1}^{V_{0}, V_{1}}\left(e_{1}^{1}\right), e_{1}\right\rangle-4 \bar{\beta}^{2}\left\langle A_{1}^{V_{r}, V_{r+1}} \circ \cdots \circ A_{1}^{V_{0}, V_{1}}\left(e_{1}^{1}\right), J e_{1}^{1}\right\rangle \\
& =\frac{s^{2}}{\bar{\beta}}\left\langle A_{r}^{V_{r}, V_{r+1}} \circ \cdots \circ A_{1}^{V_{0}, V_{1}}\left(J e_{1}\right), A_{\prime \prime}^{V_{0}, V_{r+1}}\left(e_{1}^{1}\right)\right\rangle \\
& +2 \bar{\beta} s\left\langle A_{,}^{V_{r}, V_{r+1}} \circ \cdots \circ A_{1}^{V_{0}, V_{1}}\left(J e_{1}\right),-\frac{\bar{s}}{\beta^{2}} A_{\prime \prime}^{V_{0}, V_{r+1}}\left(e_{1}^{1}\right)-\frac{1}{\beta} A_{\prime \prime}^{V_{0}, V_{r+1}}\left(J e_{1}\right)\right\rangle \\
& -2 s\left\langle A_{,}^{V_{r}, V_{r+1}} \circ \cdots \circ A_{,}^{V_{0}, V_{1}}\left(e_{1}^{1}\right), A_{\prime \prime}^{V_{0}, V_{r+1}}\left(e_{1}^{1}\right)\right\rangle \\
& -4 \bar{\beta}^{2}\left\langle A_{r}^{V_{r}, V_{r+1}} \circ \cdots \circ A_{1}^{V_{0}, V_{1}}\left(e_{1}^{1}\right),-\frac{\bar{s}}{\beta^{2}} A_{\prime \prime}^{V_{0}, V_{r+1}}\left(e_{1}^{1}\right)-\frac{1}{\beta} A_{\prime \prime}^{V_{0}, V_{r+1}}\left(J e_{1}\right)\right\rangle \\
& =-\frac{s^{2}}{\bar{\beta}}\left\langle A_{\varphi_{1}}^{F R}\left(J e_{1}\right), e_{1}^{1}\right\rangle+\frac{2 s^{2}}{\bar{\beta}}\left\langle A_{l_{1}}^{F R}\left(J e_{1}\right), e_{1}^{1}\right\rangle+2 s\left\langle A_{\varphi_{1}}^{F R}\left(J e_{1}\right), J e_{1}\right\rangle \\
& +2 s\left\langle A_{\varphi_{1}}^{F R}\left(e_{1}^{1}\right), e_{1}^{1}\right\rangle-4 s\left\langle A_{l_{1}}^{F R}\left(e_{1}^{1}\right), e_{1}^{1}\right\rangle-4 \bar{\beta}\left\langle A_{\varphi_{1}}^{F R}\left(e_{1}^{1}\right), J e_{1}\right\rangle \\
& =-\frac{\bar{\beta}}{c}\left(\frac{s^{2}}{\bar{\beta}^{2}} c^{2}+4 b c\right)=-\frac{\bar{\beta}}{c}\left\{\left(\operatorname{trace} A_{\iota_{1}}^{F R}\right)^{2}-4 \operatorname{det}\left(A_{l_{1}}^{F R}\right)\right\} .
\end{aligned}
$$

Thus, $\varphi_{2}$ has strong isotropy order greater than or equal to $r+1$ if and only if the equation ( $\left.\operatorname{trace} A_{\varphi_{1}}^{F R}\right)^{2}-4 \operatorname{det}\left(A_{\iota_{1}}^{F R}\right) \equiv 0$ holds.

Remark. (1) If $\left(\operatorname{trace} A_{l_{\varphi_{1}}}^{F R}\right)^{2}-4 \operatorname{det}\left(A_{l_{1}}^{F R}\right) \neq 0$, then we see that $\varphi_{2}$ has strong isotropy order $r$ and is quaternionic, and that $A_{\ell_{2}}^{F R}$ is nilpotent. However, $\varphi_{2}$ is different from $\varphi$.

(2) In the case where $\operatorname{det} A_{\iota_{1}}^{F R} \equiv 0$ and $\operatorname{trace} A_{\iota_{1}}^{F R} \neq 0, \widetilde{\varphi}_{1}$ defined by

$$
V\left(\widetilde{\varphi}_{1}\right)=V\left(\varphi_{1}\right) \ominus \operatorname{Im} A_{\prime \prime \varphi_{1}}^{F R} \oplus \operatorname{Im}\left(\left.A_{/ \prime}^{\varphi_{1}}\right|_{\operatorname{Im} A_{/ \prime \varphi_{1}}^{F R}}\right)
$$

has strong isotropy order at least $r+1$. However, $\widetilde{\varphi}_{1}$ is not quaternionic (it is quaternionic if $A_{\iota_{1}}^{F R}$ is nilpotent).

\section{HARMONIC TORI IN $\mathbf{H} P^{2}$ AND $\mathbf{H} P^{3}$}

In this section, we prove our results. Before stating the results, we give some definitions.

Definition. Let $\varphi: M \longrightarrow G_{2}\left(\mathbf{C}^{2 n}\right)$ be a harmonic map of strong isotropy order $r$ and $\left\{V_{i}\right\}$ be the harmonic sequence of the bundles for $\varphi$, where $V_{0}=V(\varphi)$. If there is some integer $k$ with $1 \leq k \leq r$ such that $\operatorname{rank} V_{k-1}=\operatorname{rank} V_{0}$ and $\operatorname{rankIm} A_{1}^{V_{k-1}, V_{k}}<\operatorname{rank} V_{0}$, then $\operatorname{rankIm} A_{1}^{\bar{V}_{k-1}, V_{k}}=1$ and $\operatorname{Im} A_{1}^{V_{k-1}, V_{k}}$ defines a harmonic map $\psi: M \longrightarrow \mathbf{C} P^{2 n-1}$. Let $\left\{V_{n}(\psi)\right\}$ be the harmonic sequence of the bundles for $\psi$, where $V_{0}(\psi)=V(\psi)$. Then, there is an anti-holomorphic line subbundle 
$F$ of $\left(V_{-1}(\psi) \oplus V(\psi)\right)^{\perp}$ such that $V(\varphi)=G^{(-k+1)}\left(V_{-1}(\psi) \oplus F\right)$. In this case, we say that $\varphi$ is obtained from $\psi$ by the extension and flag transforms. In particular, if $\varphi$ has images in $\mathbf{H} P^{n-1}$, we choose the above $F$ such that $G^{(-k+1)}\left(V_{-1}(\psi) \oplus F\right)$ is $J$-invariant. Then,

$$
V(\varphi)=G^{(-k+1)}\left(V_{-1}(\psi) \oplus F\right) \cap V_{-k}(\psi) \oplus J\left(G^{(-k+1)}\left(V_{-1}(\psi) \oplus F\right) \cap V_{-k}(\psi)\right) .
$$

In this case, we say that $\varphi$ is a quaternionic pair obtained from $\psi$ by the extension and flag transforms.

We denote by $n G$ the $n$-products of a Lie group $G$. We embed $U(2)$ into $S p(2)$ by $U(2) \ni\left(\begin{array}{ll}a & b \\ c & d\end{array}\right) \rightarrow\left(\begin{array}{cccc}a & 0 & 0 & b \\ 0 & \bar{d} & -\bar{c} & 0 \\ 0 & -\bar{b} & \bar{a} & 0 \\ c & 0 & 0 & d\end{array}\right) \in S p(2)$. Thus, $n U(2)$ may be embedded in $n S p(2)$. We need the following lemma :

Lemma 5.1. For $\zeta=\exp (2 \pi \sqrt{-1} / n)$ with $n \geq 2$, let $Q=\left(\begin{array}{cc}A & \mathbf{0} \\ \mathbf{0} & \bar{A}\end{array}\right) \in S p(n) \subset$ $U(2 n)$, where

$$
A=\left(\begin{array}{ccccc}
1 & 0 & 0 & \cdots & 0 \\
0 & \zeta & 0 & \cdots & 0 \\
0 & 0 & \zeta^{2} & 0 \cdots & 0 \\
\vdots & \vdots & \vdots & \ddots & \vdots \\
0 & \cdots & \cdots & 0 & \zeta^{n-1}
\end{array}\right) .
$$

Then, $\tau=\operatorname{Ad} Q$ defines an $n$-symmetric space structure on $S p(n) / 2 S p(1) \times$ $\left(\frac{n-2}{2}\right) U(2)$ or $S p(n) / S p(1) \times\left(\frac{n-1}{2}\right) U(2)$ according as $n$ is even or odd, in the same way as it defines an $n$-symmetric space structure on $U(2 n) / n U(2)$. Moreover, if $\varphi: M \longrightarrow U(2 n) / n U(2)$ is a primitive harmonic map the image of which is contained in $N=S p(n) / 2 S p(1) \times\left(\frac{n-2}{2}\right) U(2)$ or $N=S p(n) / S p(1) \times\left(\frac{n-1}{2}\right) U(2)$ according as $n$ is even or odd, then $\varphi$ is also a primitive harmonic map regarded as a map into $N$.

Proof. $\tau$ is an order $n$ automorphism of $U(2 n)$ and its stabilizer is conjugate to $n U(2)$. Since $\tau$ leaves $S p(n)$ invariant, it also defines an order $n$ automorphism of $S p(n)$. Its stabilizer is conjugate to $S p(n) \cap n U(2)$, which is $2 S p(1) \times\left(\frac{n-2}{2}\right) U(2)$ or $S p(1) \times\left(\frac{n-1}{2}\right) U(2)$ according as $n$ is even or odd. Let $\mathcal{G}$ and $\mathcal{G}^{\prime}$ be the Lie algebras of $U(2 n)$ and $S p(n)$, respectively. Since each eigenspace of $\tau$ for $\mathcal{G}^{\prime}$ is nothing but the restriction of the eigenspace of $\tau$ for $\mathcal{G}$ to $\mathcal{G}^{\prime}$, if the image of $\varphi$ is contained in $N$ then $\varphi$ is also a primitive harmonic map regarded as a map into $N$.

Now, we prove the following

Theorem 5.1. Let $\varphi: T^{2} \longrightarrow \mathbf{H} P^{2} \subset G_{2}\left(\mathbf{C}^{6}\right)$ be a non-superminimal harmonic map of a two-torus. Then, either $\varphi$ is a quaternionic pair obtained from a harmonic map into $\mathbf{C} P^{5}$ by the extension and flag transforms, or $\varphi$ is covered by a primitive harmonic map of finite type into $\mathbf{H} P^{2}$ or $S p(3) / S p(1) \times U(2)$ according as the isotropy order of $\varphi$ is one or two, respectively.

Proof. Let $r$ be the strong isotropy order of $\varphi$. Suppose that $\operatorname{rank} \operatorname{Im} A_{i}^{V_{i-1}, V_{i}}=$ $\operatorname{rank} V_{0}$ for $i=0,1, \cdots, r$; otherwise we see that $\varphi$ is a quaternionic pair obtained 
from a harmonic map into $\mathbf{C} P^{5}$ by the extension and flag transforms. Then, $r=$ 1,2. If $r=1$, then by Proposition $3.1 \varphi$ is a primitive harmonic map of finite type into $\mathbf{H} P^{2} \subset G_{2}\left(\mathbf{C}^{6}\right)$. If $r=2$, then we have $V_{-1}=V_{2}$, which implies that $\operatorname{det}\left(A_{1 \varphi}^{F R}\right) \neq 0$. Therefore, by Proposition $4.1 \varphi$ is covered by a primitive harmonic map $\psi$ of finite type into $U(6) / 3 U(2)$. Moreover, by Lemma 3.1 in [G], $\psi$ has its images in a totally geodesic submanifold $S p(3) / S p(1) \times U(2)$ of $U(6) / 3 U(2)$. Then, Lemma 5.1 implies the desired result.

Theorem 5.2. Let $\varphi: T^{2} \longrightarrow \mathbf{H} P^{3} \subset G_{2}\left(\mathbf{C}^{8}\right)$ be a non-superminimal harmonic map of a two-torus. If the strong isotropy order of $\varphi$ is odd, then $\varphi$ is covered by a primitive harmonic map of finite type into $\mathbf{H} P^{3}$ or $S p(4) / 2 S p(1) \times U(2)$ according as the strong isotropy order of $\varphi$ is one or three. If the strong isotropy order of $\varphi$ is even, then $\varphi$ is obtained by either of the following methods:

(1) If $\operatorname{det}\left(A_{1 \varphi}^{F R}\right) \neq 0$, then $\varphi$ is covered by a primitive harmonic map of finite type into $S U(8) / S(2 U(2) \times U(4))$.

(2) If $\operatorname{det}\left(A_{1 \varphi}^{F R}\right) \equiv 0$, then either $\varphi$ is a quaternionic pair obtained from a harmonic map into $\mathbf{C} P^{7}$ by the extension and flag transforms, or $\varphi$ is obtained from $\varphi_{1}: T^{2} \longrightarrow G_{2}\left(\mathbf{C}^{8}\right)$, which has strong isotropy order 3 and satisfies $V_{-1}\left(\varphi_{1}\right)=$ $J V\left(\varphi_{1}\right)$, by the backward replacement. Moreover, $\varphi_{1}$ is obtained by either of the following methods: (2-1) $\varphi_{1}$ is covered by a primitive harmonic map of finite type into $U(8) / 4 U(2)$; (2-2) $\varphi_{1}$ is obtained by the forward replacement from some $\varphi_{2}$, which is quaternionic and has strong isotropy order 3 and is covered by a primitive harmonic map into $S p(4) / 2 S p(1) \times U(2)$; (2-3) $\varphi_{1}$ is obtained from a harmonic map into $\mathbf{C} P^{7}$ by the extension and flag transforms.

Proof. Let $r$ be the strong isotropy order of $\varphi$. Suppose that $\operatorname{rank} \operatorname{Im} A_{i}^{V_{i-1}, V_{i}}=$ $\operatorname{rank} V_{0}$ for $i=1, \cdots, r$; otherwise we see that $\varphi$ is a quaternionic pair obtained from a harmonic map into $\mathbf{C} P^{7}$ by the extension and flag transforms. Then, $r=$ $1,2,3$. If $r=1$, then by Proposition $3.1 \varphi$ is a primitive harmonic map into $\mathbf{H} P^{3} \subset G_{2}\left(\mathbf{C}^{8}\right)$. If $r=3$, then by Proposition $3.1, \varphi$ is covered by a primitive harmonic map into $U(8) / 4 U(2)$. Since $J V_{1}=V_{3}$, and $V_{0}$ and $V_{2}$ are $J$-invariant, in the same way as in Lemma 3.1 in [G] we see that $\varphi$ has its images in a totally geodesic submanifold $S p(4) / 2 S p(1) \times U(2)$ of $U(8) / 4 U(2)$. Then, Lemma 5.1 implies the result of this case. If $r=2$ and $\operatorname{det}\left(A_{\iota^{F}}^{F R}\right) \neq 0$, then by Proposition 4.1 $\varphi$ is covered by a primitive harmonic map into the flag manifold stated in the theorem. If $r=2$ and $\operatorname{det}\left(A_{\iota_{\varphi}}^{F R}\right) \equiv 0$, then by Proposition 4.2 we obtain a harmonic map $\varphi_{1}$ which has strong isotropy order 3 and satisfies $V_{-1}\left(\varphi_{1}\right)=J V\left(\varphi_{1}\right)$. If $\left(\operatorname{trace} A_{\iota_{1}}^{F R}\right)^{2}-4 \operatorname{det}\left(A_{\iota_{\varphi_{1}}}^{F R}\right) \neq 0$ and $\operatorname{det}\left(A_{\iota_{1}}^{F R}\right) \neq 0$, then by Theorem 2.2, $\varphi_{1}$ is covered by a primitive harmonic map of finite type into the flag manifold stated in the theorem. If $\left(\operatorname{trace} A_{\varphi_{1}}^{F R}\right)^{2}-4 \operatorname{det}\left(A_{\varphi_{1}}^{F R}\right) \equiv 0$, then by Proposition 4.2 we obtain a harmonic map $\varphi_{2}$ which is quaternionic and has strong isotropy order 3 . By the above argument, we see that $\varphi_{2}$ is covered by a primitive harmonic map of finite type into $S p(4) / 2 S p(1) \times U(2)$. Finally, if $\operatorname{det}\left(A_{l_{1}}^{F R}\right) \equiv 0$ and $\operatorname{trace}\left(A_{\varphi_{1}}^{F R}\right) \neq 0$, then we see that the harmonic sequence of $\varphi_{1}$ is reducible, that is, $\varphi_{1}$ is reduced to a harmonic map into $\mathbf{C} P^{7}$. Hence, $\varphi_{1}$ is obtained from a harmonic map into $\mathbf{C} P^{7}$ by the extension anf flag transforms.

Remark. To classify all harmonic two-tori in $\mathbf{H} P^{n-1}$, we must analyze the class of harmonic maps $\varphi: T^{2} \longrightarrow G_{2}\left(\mathbf{C}^{2 n}\right)$ which have the properties that $\operatorname{det}\left(A_{{ }^{\prime}}^{F R}\right) \equiv 0$, $\operatorname{trace}\left(A_{/ \varphi}^{F R}\right) \neq 0$ and $V_{-1}(\varphi)=J V(\varphi)$. 


\section{REFERENCES}

[Ba-W] A. Bahy-El-Dien and J. C. Wood, The explicit construction of all harmonic two-spheres in quaternionic projective spaces, Proc. London Math. Soc. 62 (1991), 202-224. MR 91k:58021

[B] F. E. Burstall, Harmonic tori in spheres and complex projective spaces, preprint.

[B-F-P-P] F. E. Burstall, D. Ferus, F. Pedit, and U. Pinkall, Harmonic tori in symmetric spaces and commuting Hamiltonian systems on loop algebras, Ann. of Math. 138 (1993), 173-212. MR 94m:58057

[B-P-W] J. Bolton, F. Pedit, and L. Woodward, Minimal surfaces and the affine Toda field model, J. Reine Angew. Math. (to appear).

[B-P] F. E. Burstall and F. Pedit, Harmonic maps via Adler-Kostant-Symes theory, Harmonic maps and Integrable Systems (A.P. Fordy and J.C. Wood, eds.), Aspects of Mathematics E23, Vieweg, 1994, pp. 221-272. CMP 94:09

[B-R] F. E. Burstall and J. H. Rawnsley, Twistor theory for Riemannian symmetric spaces, Lect. Notes in Math. 1424, Springer-Verlag, Berlin-Heidelberg-NewYork-Tokyo, 1990. MR 91m:58039

[B-W] F. E. Burstall and J. C. Wood, The construction of harmonic maps into complex Grassmannians, J. Differential Geom. 23 (1986)), 255-297. MR 88i:58038

[F-P-P-S] D. Ferus, F. Pedit, U. Pinkall, and I. Sterling, Minimal tori in $S^{4}$, J. Reine. Angew. Math. 429 (1992), 1-47. MR 93h:53008

[G] J. F. Glazebrook, The construction of a class of harmonic maps to quaternionic projective spaces, J. London Math. Soc. 30 (1984), 151-159. MR 86h:58041

$[\mathrm{H}] \quad$ N. J. Hitchin, Harmonic maps from a 2-torus to the 3-sphere, J. Differential Geom. 31 (1990), 627-710. MR 91d:58050

[K] O. Kowalski, Generalized symmetric spaces, Lect. Notes in Math. 805, Springer-Verlag, Berlin-Heidelberg-NewYork, 1980. MR 83d:53036

[U] S. Udagawa, Harmonic maps from a two-torus into a complex Grassmann manifold, International J. Math. 6 (1995), 447-459. CMP 95:11

[Wo] J. G. Wolfson, Harmonic sequences and harmonic maps of surfaces into complex Grassmann manifolds, J. Differential Geom. 27 (1988), 161-178. MR 89c:58031

[W] J. C. Wood, The explicit construction and parametrization of all harmonic maps from the two-sphere to a complex Grassmannians, J. Reine Angew. Math. 386 (1988), 1-31. MR 89f:58045; MR 90b:58054

Department of Mathematics, School of Medicine, Nihon University, Itabashi, Tokyo 173, JAPAN

E-mail address: h01217@sinet.ad.jp 